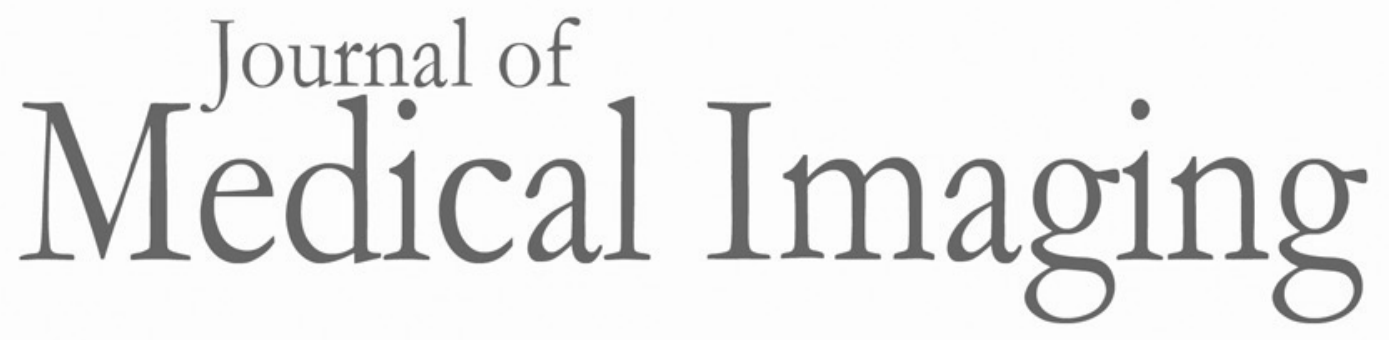

\title{
Initial evaluation of three- dimensionally printed patient-specific coronary phantoms for CT-FFR software validation
}

Lauren M. Shepard

Kelsey N. Sommer

Erin Angel

Vijay Iyer

Michael F. Wilson

Frank J. Rybicki

Dimitrios Mitsouras

Sabee Molloi

Ciprian N. Ionita 


\title{
Initial evaluation of three-dimensionally printed patient-specific coronary phantoms for CT-FFR software validation
}

\author{
Lauren M. Shepard, ${ }^{a, b}$ Kelsey N. Sommer, ${ }^{\text {a,b }}$ Erin Angel, ${ }^{c}$ Vijay lyer, ${ }^{d}$ Michael F. Wilson, ${ }^{d}$ Frank J. Rybicki, \\ Dimitrios Mitsouras, ${ }^{e}$ Sabee Molloi, ${ }^{f}$ and Ciprian N. Ionita ${ }^{a, b, g, *}$ \\ aUniversity at Buffalo, University Department of Biomedical Engineering, Buffalo, New York, United States \\ ${ }^{b}$ Canon Stroke and Vascular Research Center, Buffalo, New York, United States \\ 'Canon Medical Systems USA, Tustin, California, United States \\ dUniversity at Buffalo Medicine, Interventional Cardiology, UBMD, Buffalo, New York, United States \\ eUniversity of Ottawa, Ottawa Hospital Research Institute and the Department of Radiology, Ottawa, Canada \\ fUniversity of California Irvine, University Department of Radiological Sciences, Irvine, California, United States \\ gUniversity at Buffalo, University Department of Neurosurgery, Buffalo, New York, United States
}

\begin{abstract}
We developed three-dimensionally (3D) printed patient-specific coronary phantoms that are capable of sustaining physiological flow and pressure conditions. We assessed the accuracy of these phantoms from coronary CT acquisition, benchtop experimentation, and CT-FFR software. Five patients with coronary artery disease underwent 320-detector row coronary CT angiography (CCTA) (Aquilion ONE, Canon Medical Systems) and a catheter lab procedure to measure fractional flow reserve (FFR). The aortic root and three main coronary arteries were segmented (Vitrea, Vital Images) and 3D printed (Eden 260V, Stratasys). Phantoms were connected into a pulsatile flow loop, which replicated physiological flow and pressure gradients. Contrast was introduced and the phantoms were scanned using the same CT scanner model and CCTA protocol as used for the patients. Image data from the phantoms were input to a CT-FFR research software (Canon Medical Systems) and compared to those derived from the clinical data, along with comparisons between image measurements and benchtop FFR results. Phantom diameter measurements were within $1 \mathrm{~mm}$ on average compared to patient measurements. Patient and phantom CT-FFR results had an absolute mean difference of $4.34 \%$ and Pearson correlation of 0.95 . We have demonstrated the capabilities of 3D printed patient-specific phantoms in a diagnostic software. () The Authors. Published by SPIE under a Creative Commons Attribution 4.0 Unported License. Distribution or reproduction of this work in whole or in part requires full attribution of the original publication, including its DOI. [DOI: 10.1117/1.JMI.6.2.021603]
\end{abstract}

Key words: patient specific 3D printed phantoms; CT-FFR; coronary phantoms; blood flow simulations; coronary CT angiography. Paper 18173SSRR received Aug. 15, 2018; accepted for publication Feb. 19, 2019; published online Mar. 12, 2019.

\section{Introduction}

Coronary CT angiography (CCTA) is currently appropriate for imaging patients with defined risk factors for coronary artery disease (CAD), one of the leading causes of death in the world. ${ }^{1}$ The high negative predictive value makes CT useful for many patient cohorts, ${ }^{1,2}$ but the exam remains limited for intermediate risk patients and those with stable CAD. For intermediate risk patients, more accurate noninvasive methods are needed to determine the hemodynamic significance of CAD. The current reference standard for assessing hemodynamic significance for CAD is interventional fractional flow reserve (FFR), which determines CAD severity via hemodynamic significance. ${ }^{3}$ This technique has a cutoff value of FFR $<0.8$ for treatment; however, for FFR values in the range of 0.75 to 0.85 , the certainty of repeating the same measurement is $<80 \%$, and it is suggested for patients within this range to undergo an additional diagnostic method. ${ }^{4,5}$ There are also risks associated with this diagnostic method, such as radiation dose, traumatic injury to the coronary wall at the time of catheterization, and ischemia from plaque dislodgement. ${ }^{6,7}$ As such,

*Address all correspondence to Ciprian N. Ionita, E-mail: cnionita@ buffalo.edu noninvasive diagnostic tools have been developed for CAD risk assessment. ${ }^{8}$ Referred to as CT-FFR, these methods utilize CT angiography images and computational fluid dynamic methods to estimate the flow conditions in the coronaries, potentially bypassing the endovascular procedure and associated risks for diagnosis of CAD. CT-FFR methods incorporate coronary artery geometries from $\mathrm{CT}$ and simulate blood flow conditions to estimate pressure gradients and calculate FFR. ${ }^{9-11}$ Previous trials have been completed to investigate measurement of FFR from CCTA and successfully able to measure CT-FFR with a sensitivity and specificity of $84.3 \%$ and $87.9 \%$, but the CT-FFR did underestimate the disease severity. ${ }^{12}$ This underestimation can be a result of artifacts from the CT images, such as beam hardening or motion, and/or inaccuracies in the computational fluid dynamic simulations used, including boundary resistance, which cannot be measured noninvasively, leading to variations in CT-FFR results. ${ }^{13}$ There are challenges in accurately simulating blood flow conditions and replicating the elastic properties of vasculature. ${ }^{12}$ There is also the limiting factor of validating CT-FFR diagnostic software which requires large clinical trials to validate the technology. These aspects indicate the need for an accurate and repeatable validation method of testing which can be reproduced across various imaging platforms and simulation software. 
In recent years, 3D printing has provided researchers with an invaluable tool for replicating complex patient anatomy in a benchtop system. ${ }^{14}$ There are vast applications for $3 \mathrm{D}$ printing, and in particular, cardiovascular $3 \mathrm{D}$ printing ${ }^{15}$ has been recognized in several domains. Applications include surgical planning, simulating interventions, and replicating structural diseases. ${ }^{15}$ Previous research has shown the use of $3 \mathrm{D}$ printing to create coronary phantoms with stenosis; however, these phantoms were idealized and made out of rigid materials that do not replicate the compliance of vasculature. ${ }^{16}$ Recently, 3D printing has been utilized to create patient-specific coronary phantoms that have been successfully used for flow measurements. ${ }^{17-19}$ Despite a limited number of commercial polymers that are available for 3D printing, these phantoms have been demonstrated to approximately replicate the mechanical properties of vasculature. ${ }^{18}$ Although phantoms have been implemented in the last decade to simulate physiologic components, including flow control, elastic compliance, and controlled flow waveforms in specific arterial beds, this has not been done in the coronary circulation in a way that incorporates actual patient anatomy and vessel compliance. ${ }^{20}$ This project expands on the current applications of $3 \mathrm{D}$ printing to further develop cardiac phantoms with physiological flow conditions for accurate CT imaging of coronary flow. In addition, the accuracy of the development and manufacturing of these phantoms was assessed.

We have investigated the use of $3 \mathrm{D}$ printed patient-specific coronary phantoms to reproduce patient CT-FFR results, starting from the acquisition phase to benchtop flow experiments to software simulation. Using CT imaging, the $3 \mathrm{D}$ printed phantoms were successfully imaged with a CCTA protocol and implemented in a CT-FFR software. Accuracy of the phantoms compared to the patients was verified using measurements from the CCTA images and benchtop assessment of FFR. This research verified the use of $3 \mathrm{D}$ printed patient-specific phantoms as a physiologically accurate tool for use in and validation of diagnostic image-based software.

\section{Purpose}

3D printing of cardiovascular anatomy can be used for radiology applications, such as diagnostic software validation and image guided surgical training. We have developed accurate coronary tree phantoms from materials that are capable of sustaining physiological flow and pressure conditions while maintaining the stress and strain characteristics of human coronary arteries. These phantoms were used to improve coronary flow assessment and for imaging using common diagnostic procedures. This allowed for the 3D printed patient-specific phantoms to be used for the validation of a CT-FFR research software.

\section{Materials and Methods}

Patients underwent written informed consent and enrolled into our study following IRB approval. All patients underwent clinically indicated 320-detector row CCTA (Aquilion ONE, Canon Medical Systems) with $100 \mathrm{kVp}, 111 \mathrm{mAs}, 0.5 \mathrm{~mm}$ slice thickness, and a reconstructed voxel size of $0.63 \mathrm{~mm}$, isotropic. These patients then had a clinically indicated coronary catheterization that included invasive FFR measurement. Invasive FFR measurements were recorded at the Gates Vascular Institute (Buffalo, New York) at a distance of two lesion lengths below the distal end of the stenosis. Using angiography images from the endovascular procedure, we measured the distance along the vasculature where the clinicians measured FFR. This measurement distance was used to measure CT-FFR at the same location as the invasive FFR in both the patient and phantom images. Of the five patients used in this study, invasive FFR was performed on six vessels (4 LAD, 1 LCX, and 1 RCA).

\subsection{Phantom Design and Manufacturing}

The CCTA data were used to segment the aorta, left anterior descending (LAD), left circumflex (LCX), and right coronary artery (RCA) using a Vitrea workstation (Vital Images, Minnetonka, Minnesota) [Fig. 1 (angio CT)]. The segmented vasculature was exported as a stereolithography (STL) file for advanced mesh manipulation using a 3D modeling software (Autodesk Meshmixer, San Rafael, California) [Fig. 1 (segment geometry)]. The coronary vasculature was manipulated into a previously reported three branch approach, ${ }^{18}$ which simplifies and smoothes the vasculature geometry to include only the aorta and the three main coronary arteries. The minimal smoothing process was tailored to only reduce artifacts while maintaining the overall geometry of the arterial lumen using a previously reported technique. ${ }^{19}$ The vasculature geometry was also manipulated to create a vessel wall to allow flow. Access ports were created on the aorta and the three coronary arteries to allow connection of pressure sensors during flow experimentation. The distal vessel access ports are placed at approximately the location where the invasive FFR is measured. The final step in the mesh manipulation process was to append a base to the vasculature to provide stability during flow experimentation. This was completed by designing a base structure in SolidWorks (SolidWorks Corp., Waltham, Massachusetts) and importing the STL into Autodesk Meshmixer. Once in Autodesk Meshmixer, the base is aligned with the vasculature and a Boolean difference is performed on the base. This geometric function uses boundaries of the two structures to subtract the vasculature from the base, thus creating an opening for the inner lumen of the vasculature. ${ }^{21}$ Following the Boolean

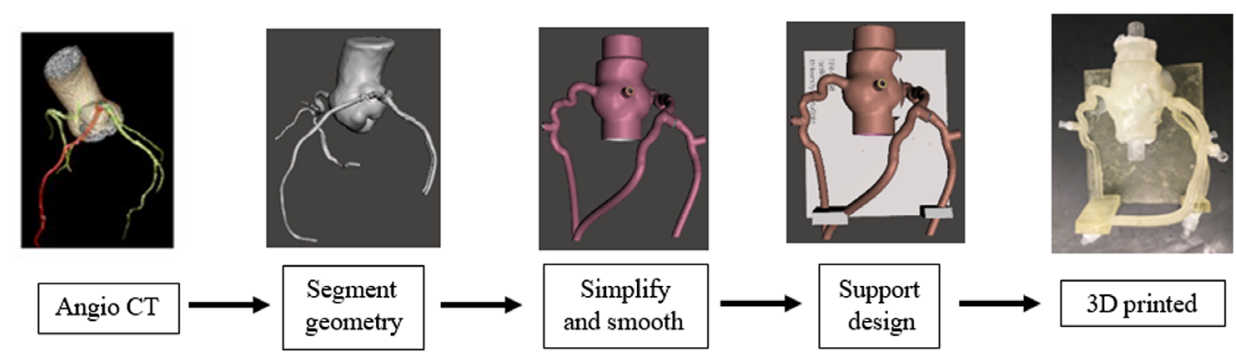

Fig. 1 Five key steps in phantom design process, starting with CT angiography images from the patient, segmentation of the desired geometry, simplifying and smoothing of vasculature, designing a support for the vasculature and appending it, then finally $3 \mathrm{D}$ printing the phantom. 
difference, the vasculature and base are combined into one structure. ${ }^{22}$ Once mesh manipulation was completed, the coronary vasculature was 3D printed using a Stratasys (Eden Prairie, Minnesota) Eden260V printer. Tango+, a soft rubber-like polymer from Stratasys, was selected for printing because the polymer mimics the compliance of vasculature. ${ }^{18}$ The process of creating the phantoms is outlined in Fig. 1, with the five main steps in the design process highlighted.

\subsection{Flow Experimentation}

Once 3D printed, each patient-specific phantom was established in a pulsatile flow loop using a CompuFlow 1000 programmable physiological flow pump (Shelley Medical Imaging Technologies, London, Ontario, Canada). This pump is capable of simulating pulsatile flow rates mimicking those seen in the coronary arteries. The user can import waveforms, allowing simulation of physiologic flow rates and waveforms, and the pump can output an ECG signal triggered by a specific point in the waveform. A $60-40 \%$ water-glycerol mixture, approximate viscosity of $3.7 \mathrm{cP}$ and approximate density of $1.1 \mathrm{~g} / \mathrm{mL},{ }^{23}$ was used with the pulsatile pump to replicate the viscosity of blood. Figure 2 shows the waveform used by the pulsatile pump to simulate physiologic conditions. Each phantom was subjected to an average flow rate of $4.3 \mathrm{~mL} / \mathrm{s}$, mimicking flow rates seen through the three main coronary arteries of $\sim 250 \mathrm{~mL} /$ min. $^{24}$
Figure 3 shows the key components of the flow loop within this study. The pulsatile pump feeds the water-glycerol mixture from a reservoir to the phantom. There are three features on the phantom: compliance chamber to dampen the flow, vessel outflow control, and pressure sensors to record the proximal and distal pressure. The compliance chamber and vessel outflow controls were used to further the accuracy of the physiological flow and pressure conditions in the phantom. First, distal resistance was generated for the coronary arteries to mimic the resistance from separate capillary beds. Previous conference proceedings from this study have shown the significant impact of distal resistance on the measured FFR values. ${ }^{25}$ Simulation of the capillary bed effect on flow is not possible with the current 3D printing technologies; while 3D printing resolution would allow creation of very fine structures, removal of support material would be very challenging. Hence, distal resistance can be simulated within reasonable ranges by controlling the diameter of the outflow tubes with mechanical clamps for each coronary artery. By adjusting the distal resistance, the flow rates and pressures in each individual artery were regulated. Second, the aorta flow and pressure were regulated using an air compliance chamber as seen in Fig. 4(a), based on a previous report. ${ }^{25}$ These two flow controls were adjusted in conjunction until pressure within the aorta reached a minimum of $80 \mathrm{mmHg}$ to ensure physiological accuracy. ${ }^{22,25,26}$ Previous in vitro studies using idealized $3 \mathrm{D}$ printed coronary phantoms have indicated

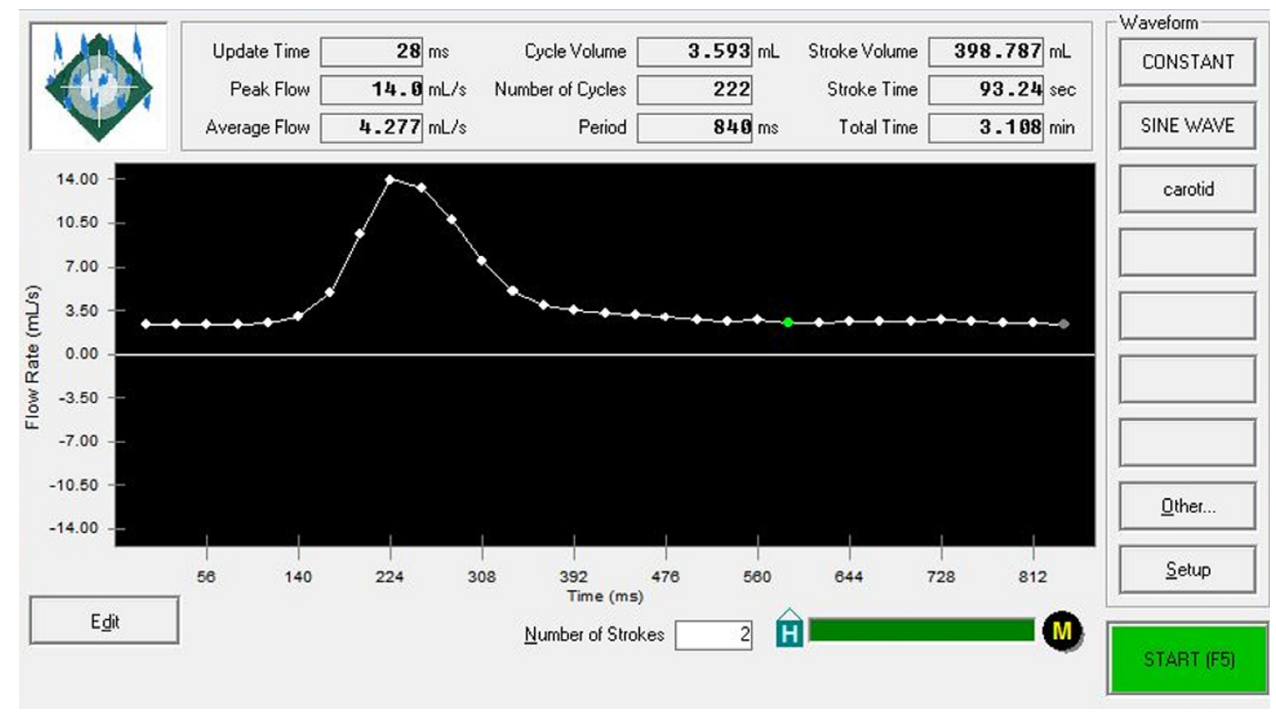

Fig. 2 Waveform used with the CompuFlow 1000 programmable pulsatile pump.

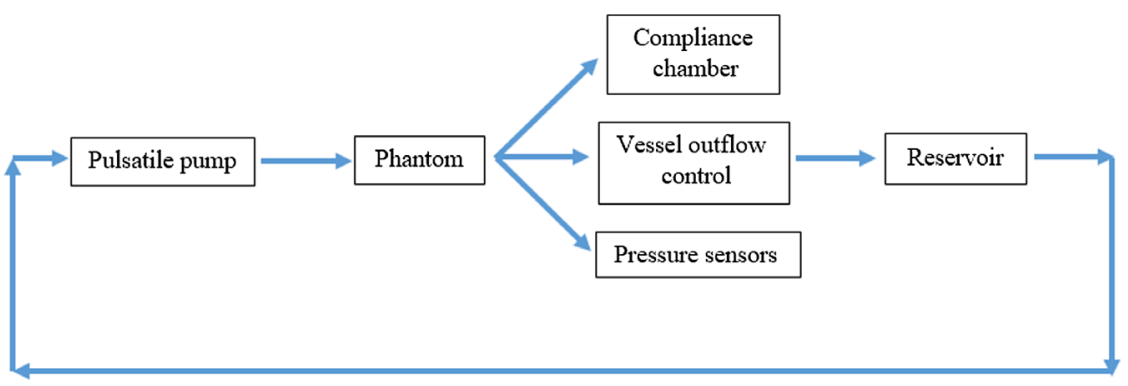

Fig. 3 Overview of key components of flow loop for experimentation. Blue arrows represent the direction of flow. 

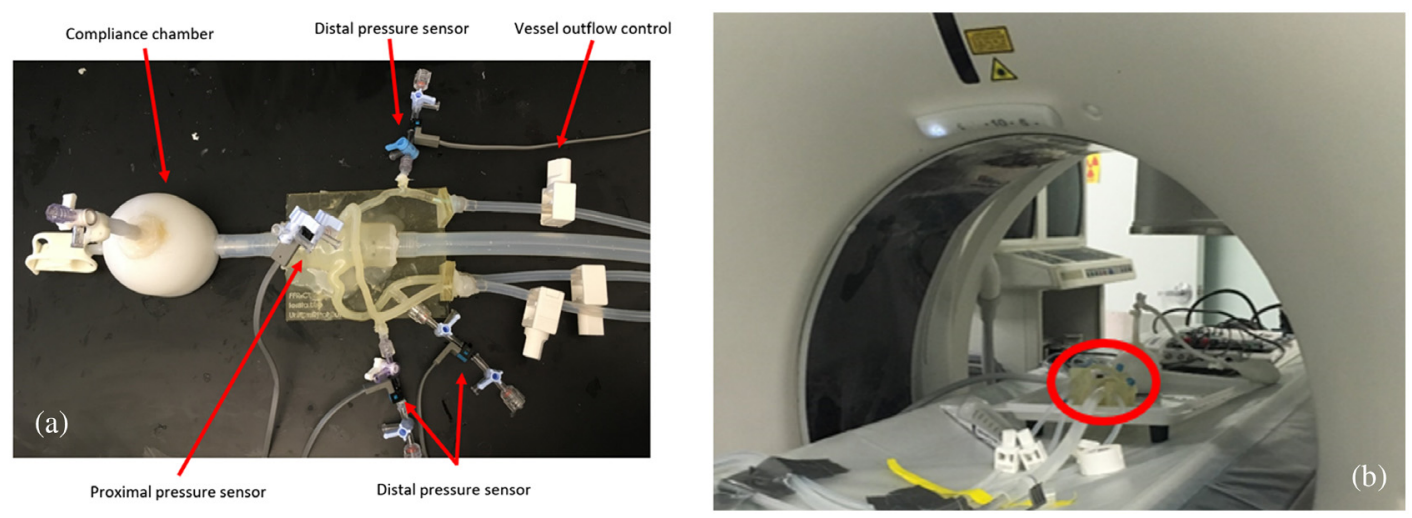

Fig. 4 Benchtop setup of 3D printed patient-specific phantoms. (a) Phantoms in established flow loop with programmable pulsatile pump with pressure sensors attached and (b) phantom, outlined in red, in Aquilion ONE scanner for CCTA scans.

a dependence on FFR with increasing aortic pressure, making this adjustment in the 3D printed phantoms a necessary step toward replicating physiological conditions. ${ }^{16}$

Figure 4(a) shows the setup of the phantoms within the flow loop and Fig. 4(b) shows the phantom setup within the CT gantry. Each phantom had pressure sensors appended to the aorta and three coronary arteries, using the access ports created during mesh manipulation, to ensure each phantom was undergoing physiologically accurate pressure conditions. These pressure values were recorded and used to calculate the "benchtop FFR" from the flow experimentation as another method to assess the accuracy of the $3 \mathrm{D}$ printed phantoms.

Once physiological flow conditions were achieved, the patient-specific phantoms were then used for CCTA testing. Each phantom underwent a similar 320-detector row CCTA (Aquilion ONE, Canon Medical Systems) to the patients, with $120 \mathrm{kVp}, 44 \mathrm{mAs}$, and $0.5 \mathrm{~mm}$ slice thickness. There was a slight variation (to better emulate the physiology) of the protocol used clinically for the five patients enrolled in this study. This variation in protocol involved a $2-\mathrm{mL}$ volume of contrast (370 mgI/mL) at a flow rate of $0.4 \mathrm{~mL} / \mathrm{s}$ followed by a saline flush being mixed in the flow loop to achieve contrast enhancement in the phantoms. To ensure similarity between the two datasets, bolus triggering with the same threshold was used for image acquisition. Thus, arteries were fully opacified in both data sets and the contrast gradient was minimized. In addition, the ECG output from the CompuFlow 1000 pulsatile pump was used to trigger the CT acquisition during the $70 \%$ to $99 \%$ R-R cycle. To replicate the CT-FFR protocol which requires four volumes to run the simulations, we also reconstructed the data for four volumes corresponding to $70 \%, 80 \%, 90 \%$, and $99 \% \mathrm{R}-\mathrm{R}$ cycle. Figure 5 shows CCTA images of both the patient (A) and the phantom (B) with three different views, for the $70 \%$ volume. Once CCTA images were collected for each phantom, the accuracy of the phantoms was assessed as described in Sec. 3.3.

\subsection{Phantom Accuracy Assessment}

The patient and phantom CCTA images were imported and segmented using Mimics Research (Materialise, Plymouth, Michigan) to perform assessment on the vasculature geometry. In order to complete the measurements, centerlines were generated for the aorta and three main vessels, LAD, LCX, and RCA. Figures 6(a) and 6(b) show a comparison of a patient and corresponding phantom geometry within Mimics, including the centerlines in red.

Once the centerlines were calculated for all three vessels, various measurements regarding the geometry were recorded at distances ranging from 10 to $100 \mathrm{~mm}$ from the ostium, using $10 \mathrm{~mm}$ increments. This was completed in all three vessels for both patients and phantoms. The parameters that were measured include: minimum diameter, maximum diameter, best fit diameter, cross-sectional area, and tortuosity. Best fit diameter is defined as the mean of all diameter measurements. Tortuosity
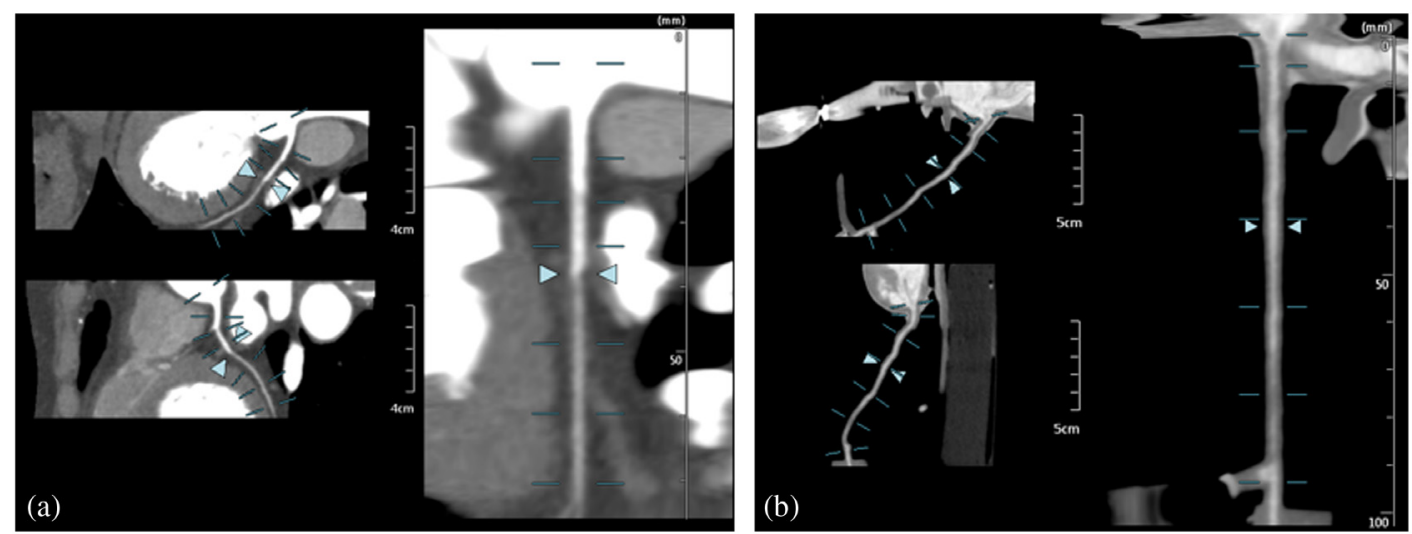

Fig. 5 CCTA images for case \#3. Patient (a) CCTA of LCX and (b) phantom CCTA images of LCX. 


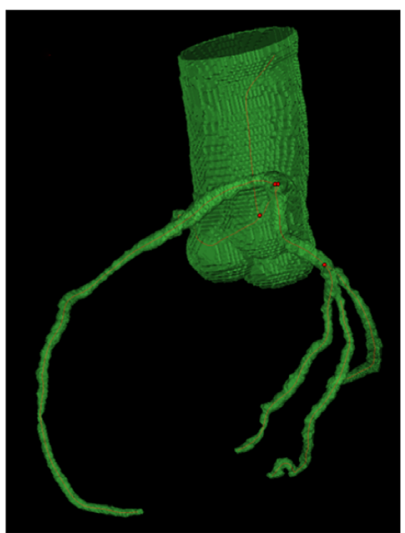

(a)

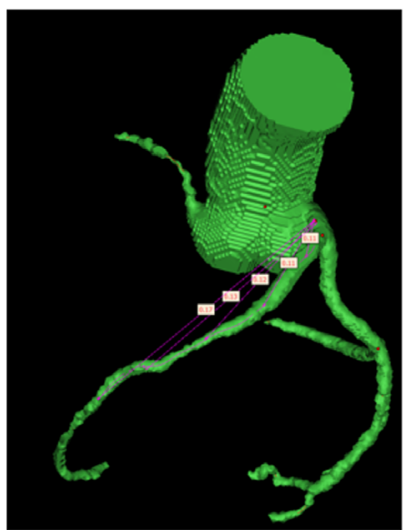

(c)

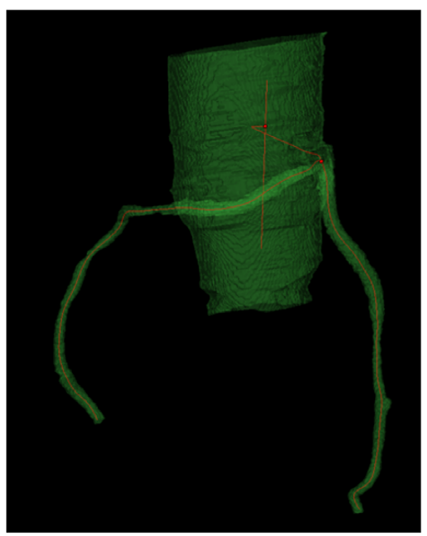

(b)

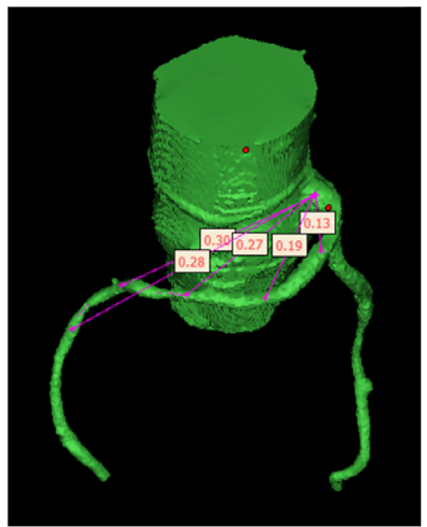

(d)
Fig. 6 (a) Segmented patient and (b) phantom CCTA images in Mimics Research, centerline shown in red. In the phantoms, only the main arteries (LAD, LCX, and RCA) were maintained. Measurement of tortuosity in (c) patient and (d) phantom.

is defined as shortest distance/vessel length and was utilized to verify the $3 \mathrm{D}$ geometry of the vasculature to ensure the soft material used in 3D printing did not deform the vasculature. Figures 6(c) and 6(d) show an example of the geometry within Mimics Research and the collection of tortuosity measurements for the LAD. Note that this patient had a mostly occluded RCA. Once the measurements were collected, the data were analyzed to determine the absolute mean differences for the phantom CCTA images from the patient CCTA images.

\subsection{CT-FFR Software}

The CT-FFR algorithm used for this research is an on-site research tool (Canon Medical Systems, Tustin, California). CT data between $70 \%$ and $99 \%$ of the R-R interval are imported into the software, ${ }^{11}$ and the phase with the least amount of motion is selected as the target phase. Once the user has selected the target phase, the software automatically calculates the centerlines and contours of the three main coronary arteries. The user then reviews the measurements and has the option to adjust centerlines and contours within the multiplanar and axial image views to ensure an accurate lumen segmentation. Once any necessary edits are made, the software utilizes multiphase acquisition and fluid structure analysis to simulate flow conditions. Details about this software have been published previously. ${ }^{10,27}$ The CT-FFR is then calculated, and the user is able to adjust the location of the CT-FFR measurement.
The CCTA images for all patients and phantoms were imported into this software and the CT-FFR was calculated following the aforementioned steps. Figure 7 shows the CT-FFR software used and the process of acquiring results.

CT-FFR results were measured at various distal locations measured from the ostium of the three main coronary arteries: LAD, LCX, and RCA. These distances ranged from 10 to $100 \mathrm{~mm}$, and $10 \mathrm{~mm}$ increments were used. In addition, the CT-FFR was recorded at a measurement distance of two lesion lengths below the distal end of the stenosis for comparison with the patient invasive FFR results. The CT-FFR results for both the patients and phantoms were quantitatively compared to determine how accurately the phantoms recreated the patient results. For this analysis, CT-FFR for the patient data was used as the reference standard.

\section{Results}

\subsection{Phantom Accuracy}

Five different parameters were measured for both patients and phantoms: minimum diameter, maximum diameter, best fit diameter, cross-sectional area, and tortuosity. As mentioned in Sec. 3.3, these measurements were collected at $10-\mathrm{mm}$ increment distances ranging from 10 to $100 \mathrm{~mm}$ from the ostium of the LAD, LCX, and RCA. Figures 8(a)-8(e) show the comparison between patient and phantom CCTA images for the five different measurements. In all figures, a line of unity is included to show the ideal correlation between the patient and phantom measurements, as well as linear regression values and trendlines. Vessels with known stenosis $(n=6)$ were assessed separately from all vessels to determine the accuracy in diseased vessels specifically.

Table 1 presents the absolute mean difference and range of differences between the patient and phantom images for all five measurements at the 10 measurement locations, as well as the overall difference. Analysis is performed on all vessels collectively and stenosed vessels separately. On average, the phantom diameter measurements were within $1 \mathrm{~mm}$ of the patient images and this difference decreased when investigating only the vessels with stenosis. The cross-sectional area had a greater difference in the phantom measurements compared to the patient. And finally, the tortuosity had a very small average difference for the phantoms, verifying that despite the impact of gravity on our elastic phantoms, we are maintaining the three-dimensional geometry.

\subsection{Benchtop FFR}

Pressure measurements were collected during flow experimentation to determine the benchtop FFR, defined as the ratio of distal to proximal pressure. The benchtop FFR results have been compared to the invasive FFR as well as the CT-FFR measured on the patient and phantom images. ${ }^{22,25}$ All FFR values were measured at approximately the same location as the invasive FFR of two lesion lengths below the distal end of the stenosis. The comparison of the four FFR measurements for the phantoms used in this study is displayed in Table 2. The stenosis grade, or the percent occluded, was measured by two users in the phantoms and compared to the patients by recording the minimum diameter in the stenosed region and dividing this value by the diameter prestenosis. The average percent stenosis and standard deviation are reported in Table 2. There was an 

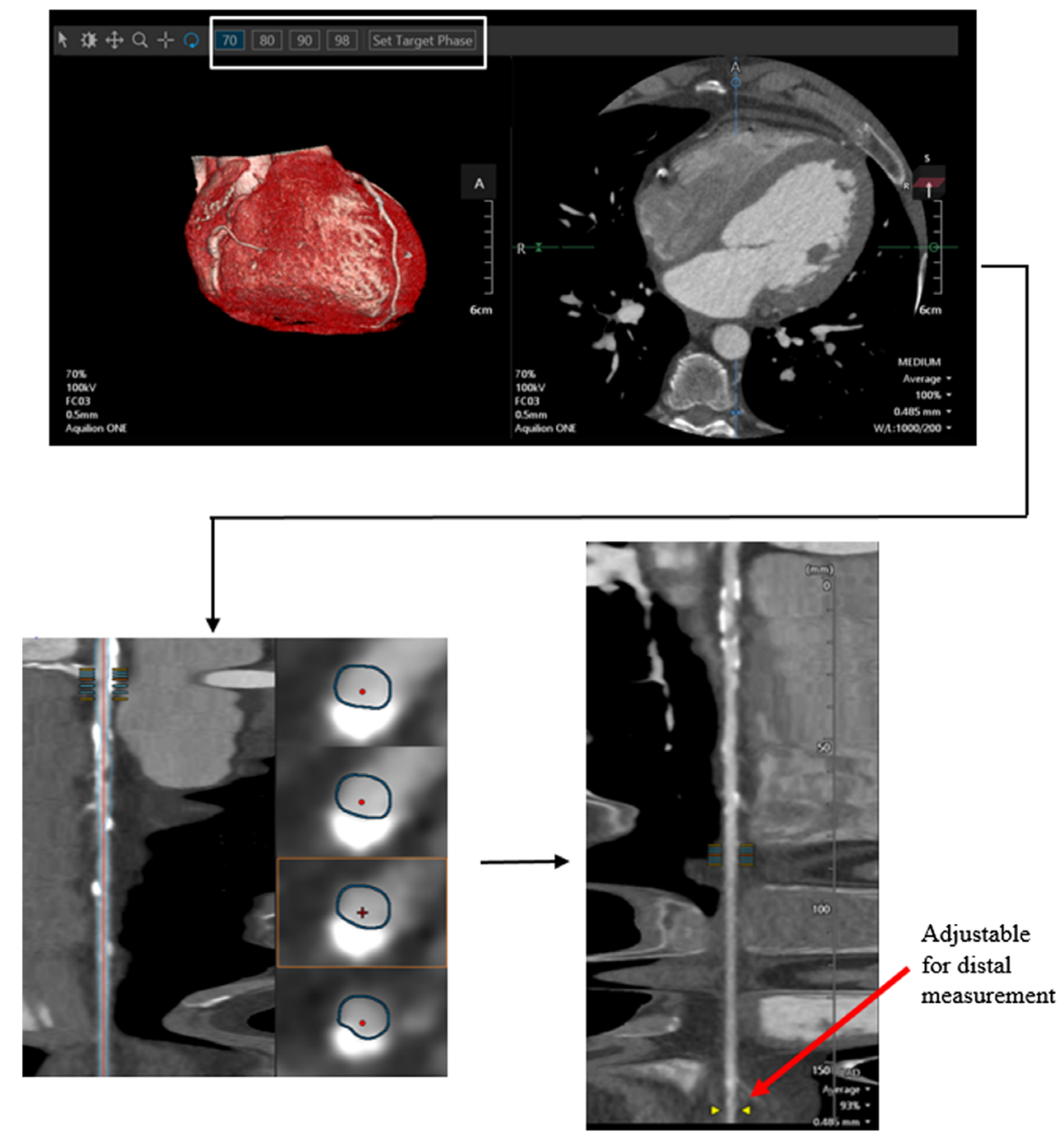

Fig. 7 CT-FFR software utilized for this research, patient data. Viewing imported images from $70 \%$ to $99 \% \mathrm{R}-\mathrm{R}$ and selecting the phase with the least amount of motion as the target phase (top image). Generation of centerline and contours (bottom left image). CT-FFR measurement with user control for distal measurement location indicated (bottom right image).



(b) Comparison of all maximum diameter measurements

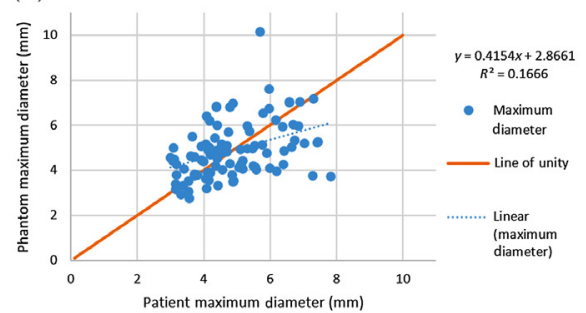
Comparison of all best fit diameter measurements
between patient and phantom CCTA image

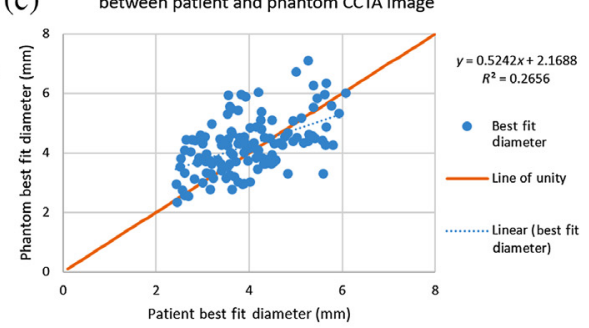

(d)

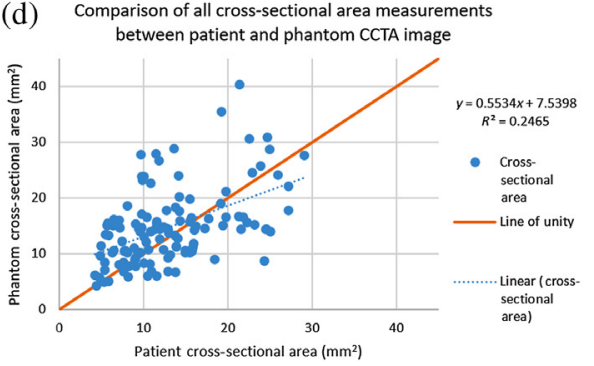

(e) Comparison of all tortuosity measurements between

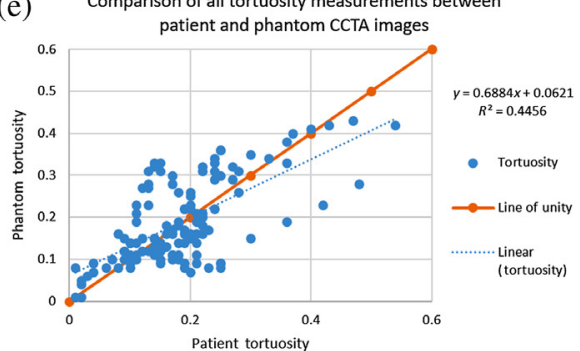

Fig. 8 Comparison of all measurements between patient and phantom images, (a) minimum diameter, (b) maximum diameter, (c) best fit diameter, (d) cross-sectional area, and (e) tortuosity. A line of unity is included in all graphs to show the ideal comparison. 
Table 1 Comparison of the absolute mean difference for the five geometric measurements along the distal vasculature at each measurement location. The absolute mean difference and range of differences were calculated for all vessels and only stenosed vessels.

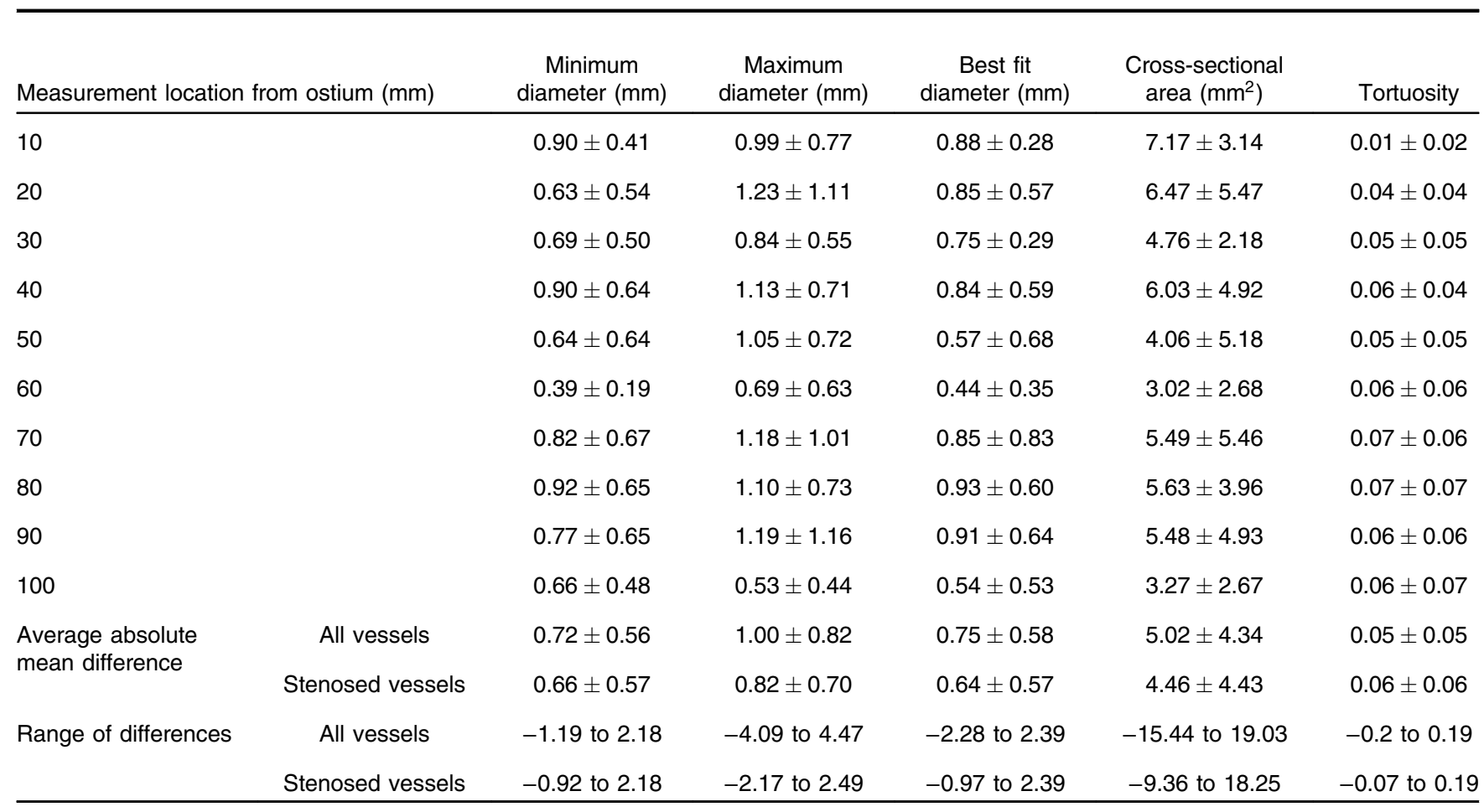

Table 2 Comparison of four measurements of FFR (benchtop FFR, invasive FFR, patient CT-FFR, and phantom CT-FFR). Case \#5 had an invasive iFR measurement (denoted *), which was converted to an FFR value. Percent stenosis measurements are included for comparison between the patients and phantoms.

\begin{tabular}{|c|c|c|c|c|c|c|c|c|}
\hline & $\begin{array}{l}\text { Stenosed } \\
\text { vessel }\end{array}$ & $\begin{array}{l}\text { Lesion length } \\
(\mathrm{mm})\end{array}$ & $\begin{array}{l}\text { Patient percent } \\
\text { stenosis (\%) }\end{array}$ & $\begin{array}{c}\text { Phantom percent } \\
\text { stenosis (\%) }\end{array}$ & Benchtop FFR & Invasive FFR & $\begin{array}{l}\text { Patient } \\
\text { CT-FFR }\end{array}$ & $\begin{array}{c}\text { Phantom } \\
\text { CT-FFR }\end{array}$ \\
\hline Case \#1 & LAD & 25 & $59.9 \pm 10$ & $53.2 \pm 0.4$ & 0.76 & 0.76 & 0.77 & 0.72 \\
\hline Case \#2 & LAD & 11.3 & $47.2 \pm 5$ & $28.1 \pm 7.2$ & 0.81 & 0.94 & 0.97 & 0.95 \\
\hline \multirow[t]{2}{*}{ Case \#3 } & LAD & 18.6 & $59.0 \pm 12$ & $56.3 \pm 12$ & 0.92 & 0.90 & 0.94 & 0.90 \\
\hline & LCX & 12.1 & $62.3 \pm 23$ & $63.2 \pm 12$ & 0.91 & 0.98 & 0.99 & 0.97 \\
\hline Case \#4 & LAD & 15.8 & $63.2 \pm 10$ & $50.7 \pm 12$ & 0.73 & 0.76 & 0.78 & 0.81 \\
\hline Case \#5 & $\mathrm{RCA}$ & 33.2 & $66.3 \pm 17$ & $38.1 \pm 10$ & 0.93 & $0.81^{*}$ & 0.92 & 0.87 \\
\hline
\end{tabular}

absolute mean difference in the minimum diameter in the stenosed region of $0.63 \mathrm{~mm}$, range 0.0 to $2.3 \mathrm{~mm}$, with a reconstructed voxel size of $0.429 \mathrm{~mm}$. Case \#5 had an iFR (instantaneous wave-free ratio) measurement instead of FFR (indicated by $*$ ), which is closely correlated to FFR as FFR = $0.68 \mathrm{iFR}+0.18 .^{28} \mathrm{We}$ used this conversion for the data analysis in this paper. Only vessels with stenosis were included as these were the vessels the clinicians recorded the invasive FFR.

Table 3 displays the Pearson correlations between the various FFR measurements. In some cases, there is significant variance when compared to the phantom results, which can be attributed to the differences in the geometry that were discussed in Sec. 4.1. While the differences in geometry were on average within $1 \mathrm{~mm}$, the difference is seen in the benchtop FFR and phantom CT-FFR results. However, with the exception of case \#4 phantom CT-FFR, all results were in agreement for treatment outcome based on the FFR threshold of 0.8 .
Table 3 Comparison of the Pearson correlation values between the four different FFR measurements (benchtop FFR, invasive FFR, patient CT-FFR, and phantom CT-FFR).

\begin{tabular}{lc}
\hline & Pearson correlation \\
\hline Invasive FFR and benchtop FFR & 0.57 \\
Invasive FFR and patient CT-FFR & 0.92 \\
Invasive FFR and phantom CT-FFR & 0.92 \\
Benchtop FFR and patient CT-FFR & 0.78 \\
Benchtop FFR and phantom CT-FFR & 0.62 \\
Patient CT-FFR and phantom CT-FFR & 0.95 \\
\hline
\end{tabular}




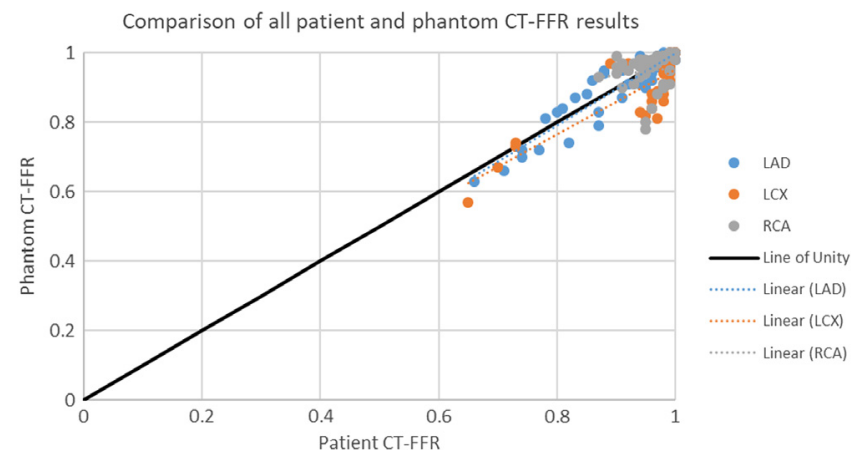

Fig. 9 Comparison of all CT-FFR results for both the phantom and the patient.

\subsection{CT-FFR Software}

As mentioned in Sec. 3.4, CT-FFR was measured at $10 \mathrm{~mm}$ increments from the ostium of each vessel, with a range from 10 to $100 \mathrm{~mm}$ in patient CT data as well as phantom CT data. A comparison was made for the overall correlation between the phantom CT-FFR and patient CT-FFR results. Figure 9 shows the comparison of all CT-FFR results for the three vessels. A line of unity was also included as the ideal result. The Pearson correlation for all patient CT-FFR and phantom CT-FFR values was 0.81 .

In addition, the percent difference was calculated for each patient to determine the accuracy of the phantoms in replicating the CT-FFR results. As shown in Sec. 4.1, the phantoms have some variation from the patient images and this is shown in the CT-FFR results. The absolute mean percent difference in the CTFFR software was calculated for the various distal measurement distances for all five patients was $4.34 \%$ and the differences ranged from $-17.89 \%$ to $10 \%$. Overall, the phantoms typically had lower CT-FFR results compared to the patients for 10 out of 14 vessels. The absolute mean percent difference calculations for each of the three main coronary vessels for the five cases are displayed in Table 4.

\section{Discussion}

We have developed a benchtop system for testing 3D printed patient-specific phantoms with physiological flow and pressure conditions. This system utilizes several features to maintain clinical flow conditions. The utilization of a pulsatile pump

Table 4 Absolute mean percent difference for patient and phantom CT-FFR results, averaged over all measurement distances for each of the three main coronary arteries (LAD, LCX, and RCA). Case \#3 has a partially occluded RCA, indicated by (n/a).

Absolute mean percent difference (\%)

\begin{tabular}{llll}
\hline Vessel & LAD & LCX & RCA \\
Case \#1 & 4.86 & 5.33 & 8.89 \\
Case \#2 & 1.94 & 8.69 & 0.64 \\
Case \#3 & 2.15 & 4.08 & $\mathrm{n} / \mathrm{a}$ \\
Case \#4 & 3.49 & 8.16 & 2.81 \\
Case \#5 & 4.42 & 2.19 & 4.47 \\
\hline
\end{tabular}

allows us to simulate flow rates and waveforms present in the coronary arteries. We utilized two flow controls that increase the accuracy of the flow conditions, the aortic flow damper and the distal mechanical clamps on the outflow tubes. The distal outflow manipulation is a critical part of the flow loop as the resistance generated by the precapillary arterioles needs to be mimicked for accurate flow experiments. Past research has demonstrated the capabilities of these phantoms for successfully measuring pressure and calculating FFR..$^{22,25}$ In addition, the previous work demonstrated the importance of flow regulation as they presented the significant variation between pressure measurements with the addition of distal resistance. ${ }^{25}$ As of now, the regulation of distal resistance is a manual process and may be different from one experiment to another, depending on main artery lumen geometry, such as diameter, tortuosity, and stenosis. This approach was implemented to replicate distal resistance due to the presence of the capillary bed and resulted in some variation of benchtop FFR measurements when compared to the gold standard (invasive FFR) as indicated in Tables 2 and 3. This method was used to bypass the current limitations of 3D printing hollow fine structures. In this setup, the differences between benchtop FFR and phantom CT-FFR were no larger than $15 \%$. Whenever a better simulation of capillary bed is needed, the modular characteristic of the 3D printed phantoms allow interfacing with more accurate phantoms that are fabricated with different technologies. ${ }^{29,30}$

Our work demonstrates that such phantoms can be scanned with commercial CT hardware for software validation starting with image acquisition, reconstruction, flow measurements, and computer simulations. Through the use of iodine contrast combined with simulated physiologic pulsatile flow, each phantom was successfully imaged using a CCTA protocol similar to the one used clinically. Visual comparisons of the images have shown that the patient geometry of the coronary vasculature is mimicked in our phantoms, with some small variations. The main difference is the differences in the aorta, which is a result of a design choice to cut the ends of the aorta to reduce printing costs. Another visual difference is the small bumps present from the sensor port additions. The pressure sensor ports may impact the computational fluid dynamics used to measure CT-FFR. In the CT-FFR software the user has the capabilities to alter the vessel contours to ensure the sensor ports are not included in the calculation. There may still be some inaccuracy in this area, but it will only impact an area of approximately $2 \mathrm{~mm}$ on the vessel. This is something that could be addressed by using other pressure sensors, such as the pressure wires used to measure FFR invasively. However, these other sensors have unknown implications on the pressure and flow conditions.

The accuracy of our 3D printed patient-specific phantoms was assessed using the segmented geometry from CCTA images and completing several measurements on the coronary arteries. We chose geometric measurements over other metrics, such as the Dice coefficient or Hausdorff distance, as these would yield inaccuracies as the phantoms are made of a soft material and gravity causes sagging of the vessels. While this is a small difference of a few millimeters, it can generate an error that is dominated by the vessel misalignment rather than the accuracy of the 3D printed phantoms. These differences were also observed in the calculation of the percent stenosis as measured by two observers in Table 2, with phantoms underestimating the stenosis severity. For the cases in which the calcium burden was low, the percent differences were moderate or negligible. However, 
in the cases with severe calcification presence, the differences were significant (case $5 \sim 40 \%$ ). These differences can be attributed to both CT artifacts in the patient data, as well as limitations in the $3 \mathrm{D}$ printing process and $3 \mathrm{D}$ printing material.

One solution is to print the phantoms with a harder material, but then we would lose the advantage of the soft material that mimics arterial compliance. Based on the geometric measurements, there were differences seen in all of the phantoms, with the various diameter measurements of the phantoms all within $1 \mathrm{~mm}$ of the patient images. However, this is a significant difference since the vessels are in the range of $2-5 \mathrm{~mm}$ in diameter. The variation in the images could be accounted by the following factors. Any discrepancies in segmentation of the patient vasculature to create the phantoms could result in the inaccurate measurements. Cardiac motion and CT artifacts from the calcification can cause segmentation discrepancies. This error would propagate throughout the results for any data regarding the phantoms. The surface roughness inherent to the $3 \mathrm{D}$ printing process might be one of the factors by allowing contrast to perfuse into the vessel wall. Another factor might be that the phantoms expanded during flow experimentation as the polymer used to create them is a soft polymer with a compliance slightly higher than that of the coronary vasculature. ${ }^{18}$ And finally, there may have been slight variations among the measurement locations along the vasculature. Care was taken to start at approximately the same location, but a slight difference in location may explain some of the differences. These are all factors to consider and they can be minimized when 3D printing patient-specific phantoms.

With the successful imaging of our phantoms under a CCTA protocol, we were able to use these images in a CT-FFR software. There were some differences between the phantom CT-FFR and patient CT-FFR results, as was expected as this is the first use of our 3D printed patient-specific coronary phantoms within a diagnostic software. This can be attributed to the variation in the geometry that was measured and reported in this paper. Previous conference reports on this research has demonstrated the use of these phantoms for simulating physiological conditions. ${ }^{22,25}$ While the phantom CT-FFR results were not in complete agreement with the patient CT-FFR results, we have demonstrated the capability of using 3D printed patient-specific phantoms within an image-based diagnostic software. The invasive FFR and patient CT-FFR show a strong positive correlation compared to the correlations with phantom results, indicating the issues arise in the phantom. Segmentation within the CT-FFR can also contribute to the variations. While the software does automatically measure centerlines and contours of the three coronary arteries, it is sometimes necessary to manually edit these features, especially in situations with high calcification and image artifacts. We are continuously improving our technique for manufacturing 3D printed patient-specific phantoms and our benchtop system, and with further experimentation, we believe these results will improve.

3D printing offers the ability to have complete control over the flow experiment, from the capability to replicate complex patient anatomy to simulating the compliance of vasculature. Through the use of $3 \mathrm{D}$ printing of patient-specific coronary phantoms and our benchtop system of flow experimentation, we were capable of implementing CCTA images of our phantom in a CT-FFR software and assessing the accuracy of these phantoms.

\section{Conclusion}

We have expanded upon previous research using 3D printed patient-specific phantoms to develop a system that utilizes these phantoms with physiological flows and pressures that are capable of being successfully imaged under CT to mimic CCTA. We have assessed the accuracy of our method of creating $3 \mathrm{D}$ printed patient-specific phantoms using the CCTA images. Our results showed that on average, the phantoms were within $1 \mathrm{~mm}$ diameter of the patient images. We have presented the accuracy of 3D printing patient-specific phantoms using the current state of the art. As the temporal and spatial resolution of CT scanners and the print resolution of $3 \mathrm{D}$ printers continue to advance, we anticipate the accuracy will continue to improve.

3D printing offers a unique solution for benchtop experimentation as patient-specific phantoms can be created that replicate the mechanical properties of the vasculature. We have demonstrated the capability of our patient-specific phantoms to undergo clinical CT protocols and be utilized within a CTFFR software. While the phantom accuracy and mechanical behavior can continue to be improved, this is an important first step toward using 3D printed patient-specific phantoms for software validation. With further improvement, we believe that $3 \mathrm{D}$ printed phantoms and this benchtop system can be used as a standard tool for validation of not only a CT-FFR software but also any image-based diagnostic software.

\section{Disclosure}

Vijay Iyer, Michael F. Wilson, Frank J. Rybicki, Dimitrious Mitsouras, and Sabee Molloi have no financial disclosures related to this research.

\section{Acknowledgments}

This research was a continuation of research published in the SPIE Medical Imaging 2018 conference proceedings, titled "CT investigation of patient-specific phantoms with coronary artery disease." ${ }^{26}$ The authors would like to acknowledge Nitant Karkhanis, Mary Lou Scholl, NP, as well as Dr. Sabee Molloi and Logan Hubbard at UC Irvine for their expert advice and contributions in developing the experimental setup. Lauren Shepard, Kelsey Sommer, and Ciprian N. Ionita were partially funded by a grant from Canon Medical Systems USA. Erin Angel is an employee of Canon Medical Systems USA. Vijay Iyer, Michael F. Wilson, Frank J. Rybicki, Dimitrious Mitsouras, and Sabee Molloi have no financial disclosures related to this research.

\section{References}

1. R. C. Cury et al., "Coronary Artery Disease-Reporting and Data System (CAD-RADS): an expert consensus document of SCCT, ACR and NASCI: endorsed by the ACC," JACC Cardiovasc. Imaging 9(9), 1099-1113 (2016).

2. F. J. Rybicki et al., "2015 ACR/ACC/AHA/AATS/ACEP/ASNC/ NASCI/SAEM/SCCT/SCMR/SCPC/SNMMI/STR/STS appropriate utilization of cardiovascular imaging in emergency department patients with chest pain: a joint document of the American College of Radiology Appropriateness Criteria Committee and the American College of Cardiology Appropriate Use Criteria Task Force," J. Am. Coll. Cardiol. 67(7), 853-879 (2016).

3. P. A. Tonino et al., "Fractional flow reserve versus angiography for guiding percutaneous coronary intervention," N. Engl. J. Med. 360(3), 213-224 (2009).

4. N. H. Pijls and J.-W. E. Sels, "Functional measurement of coronary stenosis," J. Am. Coll. Cardiol. 59(12), 1045-1057 (2012). 
5. R. Petraco et al., "Fractional flow reserve-guided revascularization: practical implications of a diagnostic gray zone and measurement variability on clinical decisions," JACC Cardiovasc. Interventions 6(3), 222-225 (2013).

6. A. I. Qureshi et al., "Prevention and treatment of thromboembolic and ischemic complications associated with endovascular procedures: Part I-pathophysiological and pharmacological features," Neurosurgery 46(6), 1344-1359 (2000).

7. A. I. Qureshi et al., "Prevention and treatment of thromboembolic and ischemic complications associated with endovascular procedures: Part II-clinical aspects and recommendations," Neurosurgery 46(6), 1360-1376 (2000).

8. C. M. Cook et al., "Diagnostic accuracy of computed tomographyderived fractional flow reserve: a systematic review," JAMA Cardiol. 2(7), 803-810 (2017).

9. J. K. Min et al., "Diagnostic accuracy of fractional flow reserve from anatomic ct angiography," JAMA 308(12), 1237-1245 (2012).

10. B. Ko et al., "Novel non-invasive CT-derived fractional flow reserve based on structural and fluid analysis (CT-FFR) for detection of functionally significant stenosis: a comparison with invasive fractional flow reserve," JACC Cardiovasc. Imaging 10(6), 663-673 (2017).

11. B. S. Ko et al., "Noninvasive CT-derived FFR based on structural and fluid analysis: a comparison with invasive FFR for detection of functionally significant stenosis," JACC Cardiovasc. Imaging 10(6), 663-673 (2017)

12. B.-K. Koo et al., "Diagnosis of ischemia-causing coronary stenoses by noninvasive fractional flow reserve computed from coronary computed tomographic angiograms: results from the prospective multicenter DISCOVER-FLOW (diagnosis of ischemia-causing stenoses obtained via noninvasive fractional flow reserve) study," J. Am. Coll. Cardiol. 58(19), 1989-1997 (2011).

13. S. Sankaran et al., "Uncertainty quantification in coronary blood flow simulations: impact of geometry, boundary conditions and blood viscosity," J. Biomech. 49(12), 2540-2547 (2016).

14. L. Chepelev et al., "Radiological Society of North America (RSNA) 3D printing Special Interest Group (SIG): guidelines for medical 3D printing and appropriateness for clinical scenarios," 3D Print. Med. 4(1), 11 (2018).

15. A. A. Giannopoulos et al., "Applications of 3D printing in cardiovascular diseases," Nat. Rev. Cardiol. 13(12), 701-718 (2016).

16. K. K. Kolli et al., "Effect of varying hemodynamic and vascular conditions on fractional flow reserve: an in vitro study," J. Am. Heart Assoc. 5(7), e003634 (2016).

17. M. Russ et al., "Treatment planning for image-guided neuro-vascular interventions using patient-specific 3D printed phantoms," Proc. SPIE 9417, 941726 (2015).

18. K. Sommer et al., "Design optimization for accurate flow simulations in 3D printed vascular phantoms derived from computed tomography angiography," Proc. SPIE 10138, 101380R (2017).

19. C. N. Ionita et al., "Challenges and limitations of patient-specific vascular phantom fabrication using 3D polyjet printing," Proc. SPIE 9038, 90380M (2014)

20. C. Ionita et al., "TU-H-CAMPUS-IeP2-03: development of 3D printed coronary phantoms for in-vitro CT-FFR validation using data from 320-detector row coronary CT angiography," Med. Phys. 43(6Part37), 3781-3781 (2016).

21. A. A. Requicha and H. B. Voelcker, "Boolean operations in solid modeling: boundary evaluation and merging algorithms," Proc. IEEE 73(1), 30-44 (1985).

22. K. N. Sommer et al., "3D printed cardiovascular patient specific phantoms used for clinical validation of a CT-derived FFR diagnostic software," Proc. SPIE $10578105780 \mathrm{~J}$ (2018).

23. G. Producers, A Physical Properties of Glycerine and Its Solutions, Glycerine Producers' Association, New York (1963).

24. T. Ramanathan and H. Skinner, "Coronary blood flow," Continuing Educ. Anaesth. Crit. Care Pain 5(2), 61-64 (2005).

25. L. Shepard et al., "Initial simulated FFR investigation using flow measurements in patient-specific 3D printed coronary phantoms," Proc. SPIE 10138, 101380S (2017).

26. L. M. Shepard et al., "CT investigation of patient-specific phantoms with coronary artery disease," Proc. SPIE 10573, 105731V (2018).

27. A. R. Ihdayhid et al., "Performance of computed tomography-derived fractional flow reserve using reduced-order modelling and static computed tomography stress myocardial perfusion imaging for detection of haemodynamically significant coronary stenosis," Eur. Heart J. Cardiovasc. Imaging 19(11), 1234-1243 (2018).

28. H. Matsuo, Y. Kawase, and I. Kawamura, "FFR and iFR," Ann. Nucl. Cardiol. 3(1), 53-60 (2017).

29. R. P. Wood et al., "Initial testing of a $3 D$ printed perfusion phantom using digital subtraction angiography," Proc. SPIE, 9417, 94170V (2015).

30. R. Eriksson et al., "A microcirculation phantom for performance testing of blood perfusion measurement equipment," Eur. J. Ultrasound 2(1), 65-75 (1995).

Lauren M. Shepard is a second-year PhD student in the group of Dr. Ciprian N. Ionita at the University at Buffalo, Canon Stroke and Vascular Research Center. This group has been a very active participant at SPIE Medical Imaging for the last 20 years with nearly 100 presentations and posters and has received various awards for scientific achievements. She has given presentations at four international conferences, including SPIE Medical Imaging and the RSNA annual meeting.

Kelsey $\mathbf{N}$. Sommer is a graduate student in the group of Dr. Ciprian N. Ionita at the University at Buffalo, Canon Stroke and Vascular Research Center. She has given presentations at four international conferences, including SPIE Medical Imaging and the RSNA annual meeting, and earned a certificate of merit at the latter.

Erin Angel is the senior manager of Clinical Collaborations at Canon Medical Systems USA, Inc. She manages research collaborations with academic and clinical institutions with a goal of clinical translation of medical imaging technologies. Her work focuses primarily on medical imaging in the areas of radiation exposure, image quality, and quantitative image analysis. She received her doctorate and master's degrees in biomedical physics from UCLA. She is actively involved in several professional organizations.

Vijay lyer is a cardiologist in Buffalo, New York, USA. He received his medical degree from the Grant Medical College at the Maharashtra University of Health Sciences and his doctorate degree from Drexel University. He is an associate professor of medicine at the University at Buffalo and the director of Structural Heart Interventions at the Gates Vascular Institute.

Michael F. Wilson is a cardiologist in Buffalo, New York, USA. He received his medical degree from Perelman School of Medicine at the University of Pennsylvania and has been in practice for more than 60 years. He is a professor emeritus of medicine at the University at Buffalo and the medical director of Cardiac CT and Nuclear Cardiology at Kaleida Health in Buffalo, New York, USA.

Frank J. Rybicki is a professor and chair of the Department of Radiology at the University of Ottawa and chief of medical imaging at the Ottawa Hospital. He is a chairperson for the American College of Radiology (ACR) Metrics Committee, vascular imaging specialty chair for the ACR Appropriateness Criteria, and a member of the joint American College of Cardiology (ACC)/ACR Appropriate Use Criteria Task Force Oversight Committee and ACR Radiation Dose Executive Committee.

Dimitrios Mitsouras is an associate professor in the Faculty of Medicine at the University of Ottawa. He received his doctorate degree from the Massachusetts Institute of Technology. He is a cofounder of the Applied Imaging Science Lab at Brigham and Women's Hospital.

Sabee Molloi is a professor of radiological sciences in the School of Medicine at University of California, Irvine, USA. He received his doctorate degree from the University of Wisconsin-Madison. He is the principal investigator of the Imaging Physics Laboratory at the University of California, Irvine, USA.

Ciprian N. Ionita is an assistant professor in biomedical engineering and neurosurgery at the University at Buffalo. He received his doctorate degree from the University at Buffalo. He is the director of the Endovascular Devices and Imaging Lab at the Canon Stroke and Vascular Research Center. 